Case report

\title{
Incipient primary biliary cirrhosis/autoimmune hepatitis overlap or hepatitic form of primary biliary cirrhosis: a case report Ranjana W Minz ${ }^{1}$, Seema Chhabra ${ }^{1}{ }^{*}$, Ritu Aggarwal $^{1}$, Ashim Das ${ }^{1}$,
Biman Saikia $^{1}$ and Yogesh K Chawla
}

Addresses: ${ }^{1}$ Department of Immunopathology, ${ }^{2}$ Department of Hepatology, Postgraduate Institute of Medical Education \& Research, Sector-12, Chandigarh - 160 012, India

Email: RWM - rwminz@hotmail.com; SC* - drseemachhabra@gmail.com; RA - ritu_immunopath@yahoo.co.in; AD - ashim126@gmail.com; BS - bimansaikia@hotmail.com; YKC - ykchawla@gmail.com

* Corresponding author

Published: 27 May 2009

Cases Journal 2009, 2:749| doi: |0.||86/|757-|626-2-749|

This article is available from: http://casesjournal.com/casesjournal/article/view/749।

(c) 2009 Minz et al; licensee Cases Network Ltd.

This is an Open Access article distributed under the terms of the Creative Commons Attribution License (http://creativecommons.org/licenses/by/3.0), which permits unrestricted use, distribution, and reproduction in any medium, provided the original work is properly cited.
Received: 27 January 2009

Accepted: 24 February 2009

\begin{abstract}
A 42 year old asymptomatic female detected as incipient Primary Biliary Cirrhosis/Autoimmune Hepatitis overlap during routine checkup. The biochemical profile showed evolution from a mildly deranged liver function test in 2004 along with increased erythrocyte sedimentation rate to a 4 times elevation of alkaline phosphatase in 2006 with mildly deranged alanine transaminase. Autoimmune markers demonstrable were Anti mitochondrial antibody $M_{2}$ and sp I00. Histopathology showed dual features, dominant findings were of autoimmune heptatitis. Features consistent with Primary Biliary Cirrhosis were minimal with an occasional portal tract showing paucity of bile ducts and occasional bile duct proliferation. Human leucocyte antigen DR/DQ genotype was as follows: DRBI*03, DRBI*07, DQBI*02, DQBI*04.
\end{abstract}

\section{Introduction}

Autoimmune diseases of liver are defined relatively simply by clinical, biochemical, radiologic and histopathological criteria into those characterized predominantly by hepatitis (AIH) or by cholestasis (PBC) [1]. PBC \& AIH are the two main immune mediated liver diseases that lie at opposite ends of the spectrum of autoimmune liver diseases and differ remarkably with respect to pathological findings and therapeutic intervention. Some patients present with features of autoimmune liver disease that do not conform uniquely to any of the established criteria for the diagnosis of autoimmune hepatitis (AIH) or primary biliary cirrhosis (PBC). Various names, mostly overlap syndrome, have been used to describe these cases. We report a unique case of an asymptomatic 42-yr-old female detected as incipient $\mathrm{PBC} / \mathrm{AIH}$ overlap during routine annual checkup.

\section{Case presentation}

A 42-yr-old Indian female, G2P2 with 2 healthy sons, residing in Cyprus, was detected to have a raised ESR during a routine health check-up in 2000. This was the only lab investigation found abnormal in the subsequent annual check-ups. She is a non alcoholic, non smoker with 
no significant medication history or any history of blood transfusion. In 2004, again during the annual heath checkup, it was detected that in addition to an increased ESR (33 with normal range of 0-20, London Clinic), she had raised liver enzymes with ALT: 64 U/L (5-65), GGT:100 U/L (10$60)$ and alkaline phosphatase: $169 \mathrm{U} / \mathrm{L}$ (40-165). In 2005, an ultrasound of abdomen and MRCP were also performed during the annual check up. USG findings showed contracted gall bladder and acoustic shadows due to non-functioning gall bladder with a small stone. No focal lesion was seen in the liver. There was mild enlargement of the right lobe of the liver. The pancreas, kidneys and spleen were normal. Magnetic resonance cholangio-pancreatography (MRCP) showed filling defects in gall bladder due to gall stones. The bile ducts and pancreas were normal. All the liver enzymes were tested thrice in 2005 in February, April and September and at all occasions found to be significantly elevated. Patient underwent cholecystectomy in October, 2005. Peroperative liver biopsy was performed that showed only mild fatty degeneration. In 2006, the liver enzymes remained elevated, the levels were as follows: alkaline phosphatase: 244 U/L, ALT: 79 U/L, GGT: 157 U/L. Patient underwent screening for hepatitis E, A, B \& C and autoimmune markers. All the viral markers were found negative. Antinuclear antibodies were positive on autoimmune serology. Liver biopsy was reviewed again.

The liver function tests were performed in 2006 at our institute and the results were as follows: AST: $53 \mathrm{U} / \mathrm{L} \mathrm{(3-}$ 39), ALT: $68 \mathrm{U} / \mathrm{L}(2-40)$, total bilirubin: $0.6 \mathrm{mg} / \mathrm{dl}(0.2-$ $0.8 \mathrm{mg} / \mathrm{dl}$ ), alkaline phosphatase: $640 \mathrm{U} / \mathrm{L}$ (98-306). Serum iron studies and $\alpha 1$-antitrypsin levels were within normal limits. Liver biopsy was twice reviewed by expert histopathologists and showed maintained lobular architecture with a moderate chronic inflammatory cell infiltrate including some plasma cells in the portal tracts. There was a focal periportal extension of inflammatory cells into the adjoining hepatocytes. Some portal tracts showed neutrophilic infiltrate involving the bile duct epithelium. The portal tracts showed mild to moderate fibrosis with focal porto-portal bridging fibrosis. Several foci of lobular inflammation were seen with a few acidophilic and preacidophilic bodies. There was mild Kupffer cell hyperplasia and sinusoidal lymphocytic infiltration. (Figure 1) Few portal tracts were devoid of bile ducts but focal ductular proliferation was noted in some. In view of these features, a diagnosis of autoimmune hepatitis with a differential diagnosis of AIH/PBC overlap was considered in this case. The patient was thoroughly worked for autoimmune markers in the Department of Immunopathology. The presence of antinuclear antibodies (ANA), anti smooth muscle antibodies (ASMA), antimitochondrial antibodies (AMA) and liverkidney microsomal antibodies (LKM) was determined

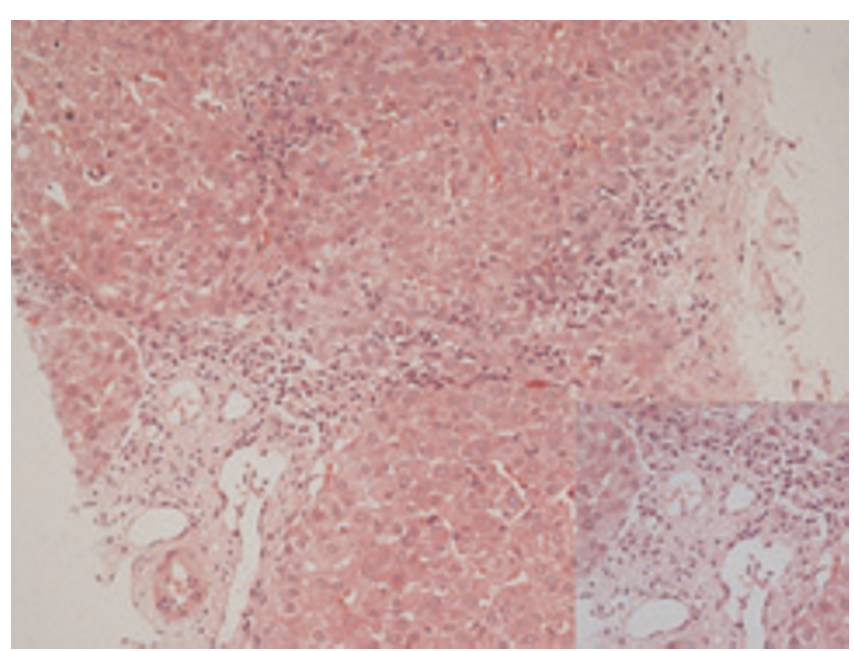

Figure I. Photomicrograph of liver biopsy showing lympho-plasmacytic infiltrate and paucity of bile ducts in portal triad, and foci of lobular inflammation (H\&E, x200). Inset showing high power view of the portal triad (H\&E, x400).

using indirect immunofluorescence on a composite of rat liver tissues (liver, kidney and stomach). Patient was found to be AMA positive $(++++)$ and ANA $(+++$, rim pattern) positive in 1:80 dilution (Figure $2 \mathrm{a}$ and $2 \mathrm{~b}$ ). ANA pattern was further analyzed using Hep- 2 cell lines that showed ++ to +++ peripheral pattern and multiple nuclear dots (MND) (Figure 3). AMA-M2 ELISA was performed in triplicate; the mean optical density was $0.769 /$ cut-off 0.388. D-tek Liver5 DOT simultaneously testing for AMAM2, LKM-1, LC-1, SLA \& F-actin was done, that again

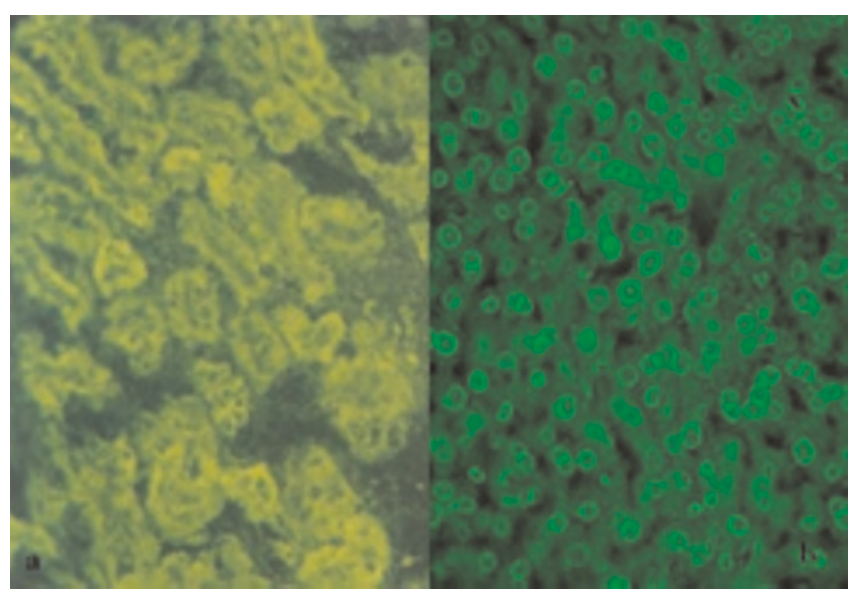

Figure 2. (a) Indirect immunofluorescence photomicrograph demonstrating strong AMA positivity on rodent kidney tissue (x200); (b) Indirect immunofluorescence photomicrograph demonstrating focal rim pattern with nuclear dots on composite rodent tissue $(x 400)$. 


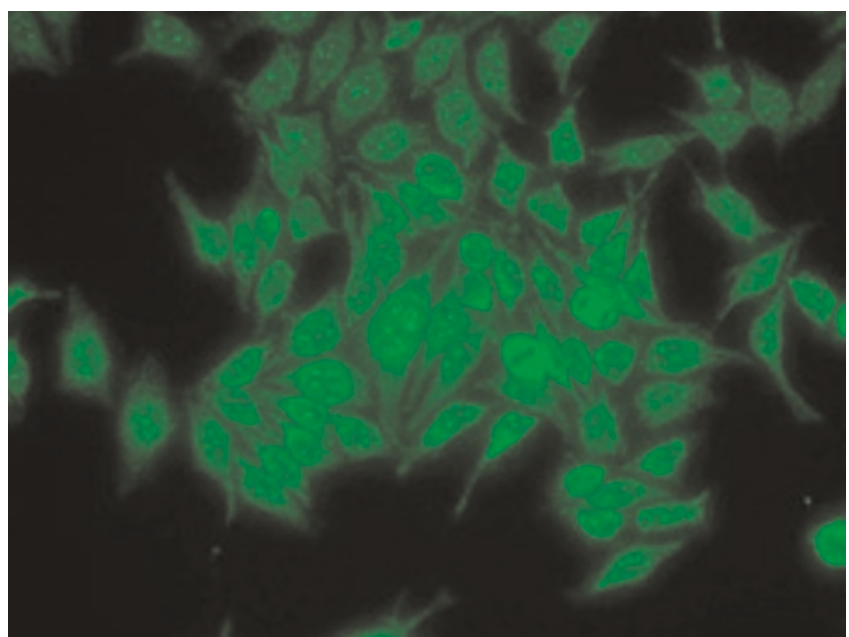

Figure 3. Indirect immunofluorescence photomicrograph of Hep2 cell lines showing typical multiple nuclear dots (MNDs) pattern (x200).

showed positivity for AMA-M2 (Figure 4a). On further testing on the Imtech LIVER LIA S blot (Human GmBH), AMA-M2 and nuclear body protein sp100 were demonstrable, whereas gp210 was negative (Figure 4b). Thus serology favored a diagnosis of primary biliary cirrhosis. To confirm the genetic susceptibility for autoimmunity, HLA DNA testing was carried out that showed a genotype of $\mathrm{DRB} 1 * 03, \mathrm{DRB} 1 * 07, \mathrm{DQB} 1 * 02, \mathrm{DQB} 1 * 04$. So based on biochemical, immunological and histopathological findings and genotype profiling, a diagnosis of incipient $\mathrm{PBC} / \mathrm{AIH}$ overlap was offered in this case. To look for concomitant autoimmune disease, testing for thyroid

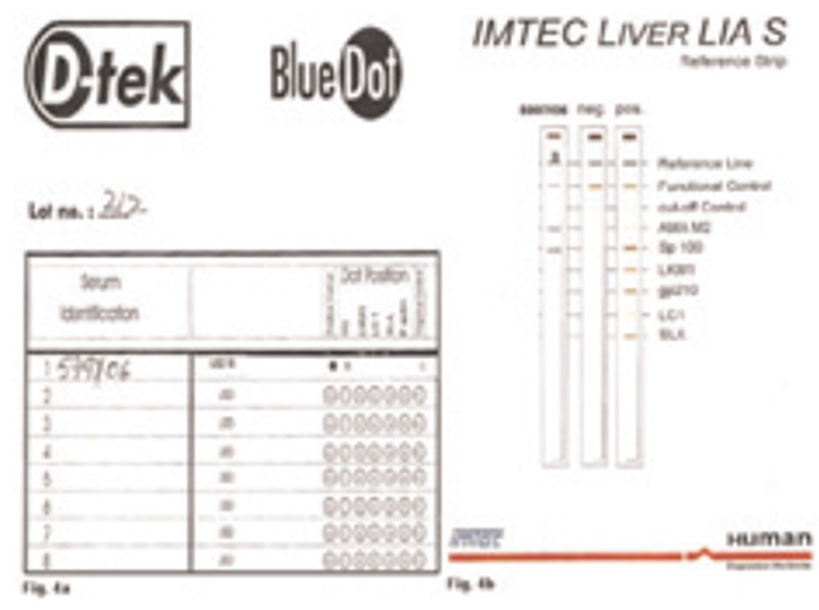

Figure 4. (a) Immunoblot with 5 antigens showing positivity for AMA $M_{2}$; (b) Immunoblot with 6 antigens showing positivity for AMA $M_{2}$ and spl00. microsomal antibodies was also done and that was negative. Patient also had gestational diabetes in last trimester of her first pregnancy in 1994. Since then blood sugar levels have remained within normal limits. Patient's mother is diabetic and also has mild arthritis.

Since September 2006 patient is taking Urosofalk. After 2 years of follow-up, she shows biochemical improvement with serologic profile remaining more or less stable on indirect immunofluorescence screening; however ELISA and immunoblot record a quantitative decline in AMA-M2 positivity.

\section{Discussion}

PBC was first described in India from our centre in 1973 as a rare entity [1]. The largest series from India describes late detection of PBC and PBC/AIH overlap (26\%) due to low intensity of screening and evaluation of liver function [2].

There is currently no consensus on what constitutes an autoimmune overlap syndrome. In true overlap syndrome, the patient has clinical, serologic and histologic characteristics of two conditions either at the same time or during the course of their illness [3]. In a study by Amrapurkar et al [4] all 6 cases of PBC/AIH overlap were detected in a late stage i.e cirrhotics and in a case report from Pune [5], the female patient presented with decompensated cirrhosis conforming to the findings of Sarin et al [2]. This index case has been detected in the incipient stage, where by using stringent diagnostic criteria (International Autoimmune Hepatitis criteria [6]: 7/15; recent simplified criteria for AIH [7]: 5/7; PBC criteria: $3 / 3$ ) the patient may be labeled as a hepatitic variant of PBC. However, the predominant features of $\mathrm{AIH}$ on histopathology are out of place in a case of PBC. Taking the presence of DR3 in this patient, it is tempting to agree with Lohse's observation that a majority of patients with overlap syndrome have the characteristic HLA haplotype of AIH namely HLA-B8, DR3 or DR4 [8]. HLA DR3 is a well known Caucasoid susceptibility gene for type $1 \mathrm{AIH}$ and its presence in our patient dictates the nature of her disease. Patients with HLA-DR3 haplotype present at an early age and enter remission less frequently during therapy.

The liver biopsy is invaluable in making a diagnosis of co-existent liver disease [9]. The index case highlights the importance of liver biopsy in making a diagnosis of $\mathrm{PBC} /$ AIH overlap.

Care must be taken while assigning AMA positive status, because AMA may not be detected by typical immunofluorescence, but may be detectable by other means such as ELISA or immunoblot using the recombinant PDC-E2 target autoantigen of M2 antibodies. Our patient was thoroughly worked up by all 3 means and finally diagnosed as AMA M2 positive. 
Antinuclear antibodies are also detectable in approximately $50 \%$ of subjects with PBC. On indirect immunofluorescence on Hep2 cells to detect ANA, two labeling patterns that predominate in PBC are punctate nuclear rim and multiple nuclear dots (MND). These patterns recognize nuclear pore membrane protein gp120 and nuclear body protein sp100 respectively. These ANAs are highly specific for PBC and detected in approximately 25\% of patients [10]. Our patient showed 5-8 nuclear dots on indirect immunofluorescence and positivity for sp100 by immunoblotting but was negative for gp120 thus providing another serologic evidence for PBC.

The recognition of autoimmune overlap syndrome is not only important from a classification standpoint, but it may have implications for therapy as recent studies demonstrate that PBC/AIH overlap has similar outcome as AIH [9]. A combination of ursodeoxycholic acid and corticosteroids is required in most patients to obtain a complete clinical and biochemical response.

It is also emphasized that there is need for closely following each and every patient of autoimmune liver disease with detailed serological profiling, histopathology of liver biopsy and HLA DNA DR/DQ analysis to unravel and comprehend the true $\mathrm{PBC} / \mathrm{AIH}$ overlap and distinguish it from hepatitic form of PBC and AIH. Such an approach will allow detection of the bulk of autoimmune diseases of the liver in India at as incipient/early a stage as ours, when therapy will be highly beneficial.

\section{Conclusion}

This case is presented to highlight that a thorough serological, biochemical and histopathological workup along with DNA DR/DQ profiling is essential to diagnose $\mathrm{PBC} / \mathrm{AIH}$ overlap which is hypothesized to be in incipient stage.

\section{Abbreviations \\ AIH, Autoimmune hepatitis; PBC, Primary biliary cirrho- sis; ESR, erythrocyte sedimentation rate; MRCP, Magnetic resonance cholangio-pancreatography; AST, Aspartate aminotransferase; ALT, Alanine aminotransferase; ANA Antinuclear antibodies; ASMA, Anti smooth muscle antibodies, AMA, Antimitochondrial antibodies, LKM, Liver-kidney microsomal antibodies; HLA DNA, Human leukocyte antigen-Deoxyribonucleic acid, ELISA, Enzyme- Linked Immunosorbent Assay.}

\section{Consent}

Written informed consent was obtained from the patient for publication of this case report and accompanying images. A copy of the written consent is available for review by the Editor-in-Chief of this journal.

\section{Competing interests}

The authors declare that they have no competing interest.

\section{Authors' contributions}

RWM has performed the immunological analysis and knitted the story of this patient. SC was the major contributor in writing the manuscript and preparing the final draft for submission. RA performed the SSP DR/DQ typing of the patient. AD reviewed the liver biopsy of the patient. BS helped with the review of the patient and photography. YKC referred the patient for serologic/HLA analysis for diagnosis and finally treated the patient. All authors read and approved the final manuscript.

\section{References}

I. Samanta AKS, Bhagwat AG, Mukherjee M, Gupta Nm, Sehgal S, Datta DV: Primary biliary cirrhosis in India. Gut 1973, 14:448-450.

2. Sarin SK, Monga R, Sandhu BS, Sharma BC, Sakhuja P, Malhotra V: Primary biliary cirrhosis in India. Hepatobilliary Pancreat Dis Int 2006, 5:105-109.

3. Poupon R, Chazouilleres O, Corpechot C, Chretien Y: Development of autoimmune hepatitis in patients with typical primary biliary cirrhosis. Hepatology 2006, 44:85-90.

4. Amarapurkar DN, Patel ND: Spectrum of autoimmune liver diseases in Western India. J of Gastroentrol and Hepatol 2007, 22:2112-2117.

5. Wagle SD, Jagtap SP, Wadia F: Primary biliary cirrhosis and autoimmune overlap syndrome. Indian J Gastroentrology 2004, 23:70-7I.

6. Alvarez F, Berg PA, Binachi FB, et al.: International autoimmune hepatitis group report: review of criteria for diagnosis of autoimmune hepatitis. J Hepatol 1993, 31:929-938.

7. Hennes EM, Zentya M, Czaja AJ, Pares A, Dalekos GM, Krawit El, et al:: Simplified criteria for the diagnosis of autoimmune hepatitis. Hepatology 2008, 48:169-176.

8. Lohse AW, Meyer zum Buschenfelde KH, Franz B, Kanzler S, Gerken $G$, Dienes HP: Characterization of the overlap syndrome of primary biliary cirrhosis and autoimmune hepatitis: evidence for it being a hepatitic form of PBC in genetically susceptible individuals. Hepatology 1999, 29:1078-1084.

9. Papamichalis PA, Zachou K, Koukoulis GK, Veloni A, Karacosta EG, Kypri I: The revised international hepatitis score in chronic liver diseases including autoimmune hepatitis/overlap syndromes and autoimmune hepatitis with concurrent other liver disorders. J Autoimmune Diseases 2007, 4:3.

10. Worman $\mathrm{HJ}$ and Courvalin Jean-Claude: Antinuclear antibodies specific for primary biliary cirrhosis. Autoimmunity Reviews 2003, 2:2II-2I7. 